\title{
Spin and pseudospin symmetries in the Dirac equation with central Coulomb potentials
}

\author{
A. S. de Castro \\ Departamento de Física e Química, Universidade Estadual Paulista, 12516-410 Guaratinguetá, São Paulo, Brazil \\ P. Alberto \\ Physics Department and Centro de Física Computacional, University of Coimbra, P-3004-516 Coimbra, Portugal
}

(Received 12 July 2012; published 28 September 2012)

\begin{abstract}
We analyze in detail the analytical solutions of the Dirac equation with scalar $S$ and vector $V$ Coulomb radial potentials near the limit of spin and pseudospin symmetries (i.e., when those potentials have the same magnitude and either the same sign or opposite signs, respectively). By performing an expansion of the relevant coefficients we also assess the perturbative nature of both symmetries and their relations to the (pseudo)spin-orbit coupling. The former analysis is made for both positive and negative energy solutions and we reproduce the relations between spin and pseudospin symmetries found before for nuclear mean-field potentials. We discuss the node structure of the radial functions and the quantum numbers of the solutions when there is spin or pseudospin symmetry, which we find to be similar to the well-known solutions of hydrogenic atoms.
\end{abstract}

DOI: 10.1103/PhysRevA.86.032122

PACS number(s): 03.65.Pm, 03.65.Ge, 31.30.jc

\section{INTRODUCTION}

Spin and pseudospin symmetries are SU(2) symmetries of a Dirac Hamiltonian with vector and scalar potentials. They are realized when the difference, $\Delta=V-S$, or the sum, $\Sigma=V+S$, are constants. These constants are zero for bound systems whose potentials go to zero at infinity. Generally, in relativistic quantum physical systems with these kinds of potentials neither of these conditions is met exactly but, in some cases, one of them can be approximately true. These symmetries may explain degeneracies in some heavy meson spectra (spin symmetry) or in single-particle energy levels in nuclei (pseudospin symmetry), when these physical systems are described by relativistic mean-field theories with scalar and vector potentials [1]. For central potentials this means that, for spin symmetry, levels with quantum numbers $(n, l, j=l-1 / 2)(n, l, j=l+1 / 2)$ are degenerate (no spin-orbit coupling), while in the case of pseudospin symmetry the degeneracy refers to the doublets $\left(n^{\prime}, l+2, j=l-1 / 2\right)(n, l, j=l+1 / 2)$, adopting, as usual, the quantum numbers of the upper component of the Dirac spinor as the quantum numbers for the full spinor. In the latter case of pseudospin symmetry, a new principal quantum number, $\tilde{n}$, and a new orbital angular momentum quantum number, $\tilde{l}$, are defined, corresponding to the quantum numbers of the lower component of the Dirac spinor, such that the doublets may be labeled by $(\tilde{n}, \tilde{l}, \tilde{j}=\tilde{l} \pm 1 / 2)$. The relations between $n^{\prime}, \tilde{n}$, and $n$ may depend on the shape of the central mean field. Most studies of spin and pseudospin symmetries are concerned with bound states, but recently it was found that the same conclusions can be applied to resonant states, namely that in conditions of pseudospin symmetry the same quantum numbers will be conserved and the pseudospin doublets would have the same energy and width [2]. However, for bound systems whose potentials go to zero at infinity, pseudospin symmetry cannot be realized, because $\Sigma=S+V$ (which must go to zero in the limit of exact pseudospin symmetry) is also the binding potential. Recently there has been a considerable interest in studying antifermions in the context of spin and pseudospin symmetries, for antinucleon systems [3-6] and antihyperons systems [7]. In this case, one cannot have exact spin symmetry (i.e., $V=S$ ) since now $-\Delta=-(V-S)$ acts as binding potential. This behavior has been related by several authors to the perturbative nature of spin and pseudospin symmetry, namely that in the case of fermions (antifermions) the pseudospin (spin) symmetry is nonperturbative for nuclear mean-field potentials [6,8-10]. Note, however, that for harmonic oscillator systems one can have exact pseudospin and spin symmetries for fermions and antifermions respectively [11].

The problem of a fermion moving in the background of a general mixing of vector and scalar Coulomb fields has been originally solved for investigating the implications of a tiny contribution of the scalar Coulomb potential to the atomic spectroscopy [12]. Recently, Zarrinkamar et al. showed that an extension of this problem including a tensor central potential of Coulomb type has analytical solutions in spin and pseudospin symmetry conditions [13]. In this paper we explore the fact that the former problem has analytical solutions for arbitrary (to a point) scalar and vector Coulomb radial potentials to examine in a detailed and explicit way, both for fermions and antifermions, the onset of pseudospin and spin symmetries and assess their perturbative nature.

This paper is organized as follows. In Sec. II we review the general solutions of the Dirac equation with spherical scalar and vector Coulomb potentials, discuss their quantum numbers and their relation with (pseudo)spin quantum numbers, and present the formulas for the energy eigenvalues. We also discuss in detail the node structure of the radial wave functions and compare them to what is known from other kind of radial mean-field potentials. In the next section we present the expansions of the energy eigenvalues for positive and negative energy solutions in terms of relevant potential parameters for spin and pseudospin symmetries and discuss the perturbative nature of those symmetries. Next we discuss the quantum numbers and extra degeneracies of the Dirac equation solutions when there is spin and pseudospin symmetry. We find that these 
are similar to the ones found in nonrelativistic hydrogenic atoms. Finally, we conclude in Sec. IV.

\section{SOLUTIONS OF THE DIRAC HAMILTONIAN WITH SCALAR AND VECTOR COULOMB POTENTIALS}

The Dirac Hamiltonian with scalar $S$ and vector $V$ potentials reads

$$
H=\boldsymbol{\alpha} \cdot \boldsymbol{p} c+\beta\left(m c^{2}+S\right)+V,
$$

where $\alpha$ and $\beta$ are the Dirac matrices in the usual representation

$$
\alpha=\left(\begin{array}{cc}
0 & \sigma \\
\sigma & 0
\end{array}\right), \quad \beta=\left(\begin{array}{cc}
I & 0 \\
0 & -I
\end{array}\right),
$$

where $\sigma$ are the Pauli matrices and $I$ is the $2 \times 2$ unit matrix. If $S$ and $V$ are of Coulomb type one has

$$
\begin{aligned}
& V=\frac{\alpha_{V}}{r} \hbar c \\
& S=\frac{\alpha_{S}}{r} \hbar c .
\end{aligned}
$$

The Hamiltonian (1) can be written in terms of the sum and difference potentials $\Sigma=V+S$ and $\Delta=V-S$ as

$$
H=\boldsymbol{\alpha} \cdot \boldsymbol{p} c+\beta m c^{2}+\frac{1}{2}(I+\beta) \Sigma+\frac{1}{2}(I-\beta) \Delta,
$$

where

$$
\begin{aligned}
& \Sigma=\frac{\alpha_{\Sigma}}{r} \hbar c, \quad \alpha_{\Sigma}=\alpha_{V}+\alpha_{S} \\
& \Delta=\frac{\alpha_{\Delta}}{r} \hbar c, \quad \alpha_{\Delta}=\alpha_{V}-\alpha_{S} .
\end{aligned}
$$

The solution of the time-independent Dirac equation

$$
H \psi=E \psi,
$$

with potentials (6) and (7) is of the form

$$
\psi=\left(\begin{array}{c}
i \frac{g_{\kappa}(r)}{r} \phi_{\kappa m_{j}}(\theta, \varphi) \\
-\frac{f_{\tilde{\kappa}}(r)}{r} \phi_{\tilde{\kappa} m_{j}}(\theta, \varphi)
\end{array}\right),
$$

where

$$
\kappa=\left\{\begin{array}{ll}
-(\ell+1) & j=\ell+\frac{1}{2} \\
\ell & j=\ell-\frac{1}{2}
\end{array},\right.
$$

$\ell$ is the orbital angular momentum of the upper component and $\tilde{\kappa}=-\kappa$. The angular functions $\phi_{\kappa m_{j}}(\theta, \varphi)$ are the spinor spherical harmonics and $g_{\kappa}(r)$ and $f_{\kappa}(r)$ are the radial wave functions for the upper and lower components of the Dirac spinor respectively. The orbital and total angular momenta quantum numbers can be obtained from $\kappa$ by $\ell=|\kappa|+$ $1 / 2(\kappa /|\kappa|-1)$ and $j=|\kappa|-1 / 2$.

The analytical bound-state solutions of Eq. (8) with the Hamiltonian (5) with the potentials (6) and (7) can be taken from Greiner and Rafelski [14], replacing the scalar and vector potentials by their sum and difference. One has for the radial wave functions, using the notation of Leviatan [15]

$$
\begin{gathered}
g_{\kappa}(r)=-A \sqrt{m c^{2}+E}\left[\left(\kappa+\eta_{2}\right) F_{1}+n_{r} F_{2}\right] \rho^{\gamma} e^{-\rho / 2} \\
f_{\kappa}(r)=A \sqrt{m c^{2}-E}\left[\left(\kappa+\eta_{2}\right) F_{1}-n_{r} F_{2}\right] \rho^{\gamma} e^{-\rho / 2}
\end{gathered}
$$

where $F_{1}={ }_{1} F_{1}\left(-n_{r}, 2 \gamma+1, \rho\right) \quad$ and $\quad F_{2}={ }_{1} F_{1}\left(-n_{r}+\right.$ $1,2 \gamma+1, \rho)$ are confluent hypergeometric functions and

$$
\begin{gathered}
\rho=2 \lambda r \\
\lambda=\frac{1}{\hbar c} \sqrt{m^{2} c^{4}-E^{2}} \\
\gamma=\sqrt{\kappa^{2}-\alpha_{\Delta} \alpha_{\Sigma}} \\
n_{r}=-\left(\gamma+\eta_{1}\right) \\
\eta_{1}=\frac{1}{2 \lambda \hbar c}\left[\alpha_{\Sigma}\left(E+m c^{2}\right)+\alpha_{\Delta}\left(E-m c^{2}\right)\right] \\
\eta_{2}=\frac{1}{2 \lambda \hbar c}\left[\alpha_{\Sigma}\left(E+m c^{2}\right)-\alpha_{\Delta}\left(E-m c^{2}\right)\right] .
\end{gathered}
$$

The integers $n_{r}=0,1,2, \ldots$ are the quantum numbers defined by the quantization condition (16) required in order that the radial functions $g_{\kappa}(r)$ and $f_{\kappa}(r)$ be normalizable, and the respective normalization condition

$$
\int_{0}^{\infty}\left[g_{\kappa}^{2}(r)+f_{\kappa}^{2}(r)\right] d r=1
$$

determines the constant $A$.

One usually defines the principal quantum number as $n=$ $n_{r}+|\kappa| n=1,2, \ldots$ so that one can write (16) as

$$
\eta_{1}=-n+|\kappa|-\gamma=-\xi
$$

Since $\xi=n_{r}+\gamma$ is positive definite, this last relation, together with the condition that $\lambda$, given by (14), must be real to have bound solutions, yields the following constraints to the energies and coefficients $\alpha_{\Delta}$ and $\alpha_{\Sigma}$ :

$$
\begin{gathered}
|E|<m c^{2} \\
\alpha_{\Sigma}\left(E+m c^{2}\right)+\alpha_{\Delta}\left(E-m c^{2}\right)<0 .
\end{gathered}
$$

This last equation can be rewritten as

$$
\alpha_{\Sigma}<\alpha_{\Delta} \frac{m c^{2}-E}{m c^{2}+E} .
$$

Another condition comes from the requirement that $\gamma$ be real. One has

$$
\alpha_{\Sigma} \alpha_{\Delta}<\kappa^{2}
$$

This condition and (23) imply that there is no bound-state solution if $\alpha_{\Sigma}>0$ and $\alpha_{\Delta}<0$. If $\alpha_{\Sigma}$ and $\alpha_{\Delta}$ have the same sign both conditions (23) and (24) constrain the values of the strengths of the potentials. When $\alpha_{\Sigma}<0$ and $\alpha_{\Delta}>0$ those conditions do not restrict the strength of these potentials. On the other hand, since one would also like to have $\alpha_{V}<0$ (standard attractive Coulomb potential) for positive energy states, then one must have $\left|\alpha_{\Sigma}\right|>\left|\alpha_{\Delta}\right|$ if the potentials have different signs. Thus, in the examples presented below, we shall use values for the strengths of the Coulomb potentials such that $\alpha_{\Sigma}<0, \alpha_{\Delta}>0$ and $-\alpha_{\Sigma}>\alpha_{\Delta}$. 
From (16), (17), and (18) and one can derive the following useful relations:

$$
\eta_{2}^{2}=\eta_{1}^{2}+\alpha_{\Delta} \alpha_{\Sigma}=n_{r}\left(n_{r}+2 \gamma\right)+\kappa^{2} .
$$

From Eqs. (14), (17), and (20) one can calculate the eigenenergies. One gets two types of solutions, denoted by $E^{ \pm}$

$$
E_{n_{r}, \kappa}^{ \pm}=m c^{2} \frac{\alpha_{\Delta}^{2}-\alpha_{\Sigma}^{2} \pm 4 \xi \sqrt{\xi^{2}+\alpha_{\Delta} \alpha_{\Sigma}}}{\left(\alpha_{\Delta}+\alpha_{\Sigma}\right)^{2}+4 \xi^{2}} .
$$

The dependence on $n_{r}$ and $\kappa$ comes through $\xi=n_{r}+\gamma=$ $n_{r}+\sqrt{\kappa^{2}-\alpha_{\Delta} \alpha_{\Sigma}}=n-|\kappa|+\sqrt{\kappa^{2}-\alpha_{\Delta} \alpha_{\Sigma}}$.

The signs were chosen such that $E^{+}$is a positive energy state and $E^{-}$is a negative energy state when either $\alpha_{\Delta}$ or $\alpha_{\Sigma}$ are very small (see the following section). Since charge conjugation (c.c.) produces the changes $V \rightarrow-V$ and $S \rightarrow S$, $E \rightarrow-E$, one has

$$
\begin{aligned}
\alpha_{\Delta} & \stackrel{\text { c.c. }}{\longrightarrow}-\alpha_{\Sigma} \quad \alpha_{\Sigma} \stackrel{\text { c.c. }}{\longrightarrow}-\alpha_{\Delta} \\
\xi & \left.\stackrel{\text { c.c. }}{\longrightarrow} \xi \text { (same quantum numbers } \kappa \text { and } n_{r}\right) \\
E_{n_{r}, \kappa}^{ \pm} & \stackrel{\text { c.c. }}{\longrightarrow} E_{n_{r}, \kappa}^{\mp} .
\end{aligned}
$$

This means that the whole spectrum maps into itself with respect to the charge conjugation operation and therefore contains both particle and antiparticle states. Also we note that there is a double degeneracy in the energy levels (besides the spin degeneracy), because the eigenenergies (26) only depend on the magnitude of $\kappa$ [except when $n_{r}=0$ (see the discussion below)]. As remarked by Leviatan [15] this degeneracy has to do with the fact that the Hamiltonian (1), when the scalar and vector potentials are of Coulomb type, commutes with the operator

$$
\begin{aligned}
J= & -i K \gamma^{5}\left(H-\beta m c^{2}\right)+\frac{\boldsymbol{\Sigma} \cdot \boldsymbol{r}}{r}\left(\alpha_{V} m c^{2}+\alpha_{S} H\right) \\
= & -i K \gamma^{5}\left(H-\beta m c^{2}\right)+\frac{\boldsymbol{\Sigma} \cdot \boldsymbol{r}}{r} \\
& \times\left[\left(\alpha_{\Sigma}+\alpha_{\Delta}\right) m c^{2}+\left(\alpha_{\Sigma}-\alpha_{\Delta}\right) H\right] / 2 .
\end{aligned}
$$

where

$$
\Sigma=\left(\begin{array}{ll}
\sigma & 0 \\
0 & \sigma
\end{array}\right)
$$

and $K$ is related to the $\boldsymbol{L} \cdot \boldsymbol{S}$ operator (see Sec. III) such that the spinor (9) is its eigenspinor with eigenvalue $\kappa$. This is a generalization of the Johnson-Lippmann operator $[15,16]$, which is itself a generalization of the Laplace-Runge-Lenz operator of the nonrelativistic quantum Coulomb problem to the relativistic one. Since $J$ anticommutes with $K$, the energy must depend only on $|\kappa|$.

One may check that the nonrelativistic limit is correct. If one sets $\alpha_{\Sigma}=\alpha_{\Delta}=\alpha_{V}$ and have $\alpha_{V} \ll \xi, \xi \sim n$, one gets

$$
E^{+}-m c^{2} \sim-m c^{2} \frac{\alpha_{V}^{2}}{2 n^{2}} .
$$

Given that, from (3), we may set, for the quantum system of an electron in an atom of atomic number $Z, \alpha_{V}=-Z \alpha$, where $\alpha=e^{2} /(\hbar c)$ is the fine structure constant, Eq. (31) is just the energy of the level $n$ of an electron of a hydrogenic atom with atomic number $Z$.

\section{A. Node structure of the solutions}

We discuss now the node structure of the radial wave functions (11) and (12). The solution with $n_{r}=0$ deserves a special consideration. In this case the radial functions $g_{\kappa}(r)$ and $f_{\kappa}(r)$ will reduce to

$$
\begin{gathered}
g_{\kappa}(r)=-B \sqrt{m c^{2}+E}\left(\kappa+\eta_{2}\right) \rho^{\gamma} e^{-\rho / 2} \\
f_{\kappa}(r)=B \sqrt{m c^{2}-E}\left(\kappa+\eta_{2}\right) \rho^{\gamma} e^{-\rho / 2},
\end{gathered}
$$

where $B$ is a constant. These functions have only one node at the origin. Furthermore, we may note that if $\alpha_{\Sigma}$ and $\alpha_{\Delta}$ have the same sign, $\gamma<1$, and therefore the radial wave functions $g_{\kappa}(r) / r f_{\kappa}(r) / r$, although integrable, would be singular at the origin. Another important point is that, in this case, from (25), $\eta_{2}= \pm|\kappa|$. Because of the factor $\kappa+\eta_{2}$ in Eqs. (32) and (33) a nonzero wave function cannot exist for any sign of $\kappa$. It turns out, as already remarked by Leviatan [15], that for $E^{+}$ solutions one must have $\kappa<0$ while for $E^{-}$solutions one must have $\kappa>0$, which means that for $n_{r}=0$ the degeneracy in the sign of $\kappa$ is broken.

For $n_{r} \geqslant 1, g_{\kappa}(r)$ and $f_{\kappa}(r)$ can be written as

$$
\begin{aligned}
g_{\kappa}(r)= & -A^{\prime} \sqrt{m c^{2}+E}\left[\left(\kappa+\eta_{2}\right) L_{n_{r}}^{2 \gamma}(\rho)\right. \\
& \left.+\left(2 \gamma+n_{r}\right) L_{n_{r}-1}^{2 \gamma}(\rho)\right] \rho^{\gamma} e^{-\rho / 2} \\
f_{\kappa}(r)= & A^{\prime} \sqrt{m c^{2}-E}\left[\left(\kappa+\eta_{2}\right) L_{n_{r}}^{2 \gamma}(\rho)\right. \\
& \left.-\left(2 \gamma+n_{r}\right) L_{n_{r}-1}^{2 \gamma}(\rho)\right] \rho^{\gamma} e^{-\rho / 2},
\end{aligned}
$$

where $L_{n_{r}}^{2 \gamma}(\rho)$ are the generalized Laguerre polynomials of degree $n_{r}=n-|\kappa|$ and $A^{\prime}$ is a constant. These polynomials have $n_{r}$ distinct positive zeros. These formulas suggest, then, that $g_{\kappa}(r)$ and $f_{\kappa}(r)$ may have the same number of nodes, namely $n_{r}+1\left(n_{r}=n-|\kappa|\right.$ nodes for $r>0$ plus one at $r=$ $0)$. In the following we prove that if $\alpha_{\Sigma} \alpha_{\Delta}<0$ then indeed the number of nodes of $g_{\kappa}(r), n_{g}$, and the number of nodes of $f_{\kappa}(r), n_{f}$, are both equal to $n_{r}+1$ for any sign of $\kappa$.

The zeros of generalized Laguerre polynomials of different degrees are interweaved [i.e., the $i$ th zero of $L_{n_{r}-1}^{2 \gamma}(\rho)$ is localized between the $i$ th and $(i+1)$ th zero of $\left.L_{n_{r}}^{2 \gamma}(\rho)\right]$. The value of these polynomials at $\rho=0$ is positive and increases with the polynomial degree. As a consequence, one finds that the number of zeros $n_{L}$ of the linear combination $(A \neq 0$, $B \neq 0$ )

$$
L(\rho)=A L_{n_{r}}^{2 \gamma}(\rho)+B L_{n_{r}-1}^{2 \gamma}(\rho)
$$

depends on the ratio $B / A$ in the following way:

$$
n_{L}=\left\{\begin{array}{ll}
n_{r}-1, & \frac{B}{A}<-\frac{L_{n_{r}}^{2 \gamma}(0)}{L_{n_{r}-1}^{2 \gamma}(0)} \\
n_{r}, & \frac{B}{A} \geqslant-\frac{L_{n_{r}}^{2 \gamma}(0)}{L_{n_{r}-1}^{2 \gamma}(0)}
\end{array} .\right.
$$



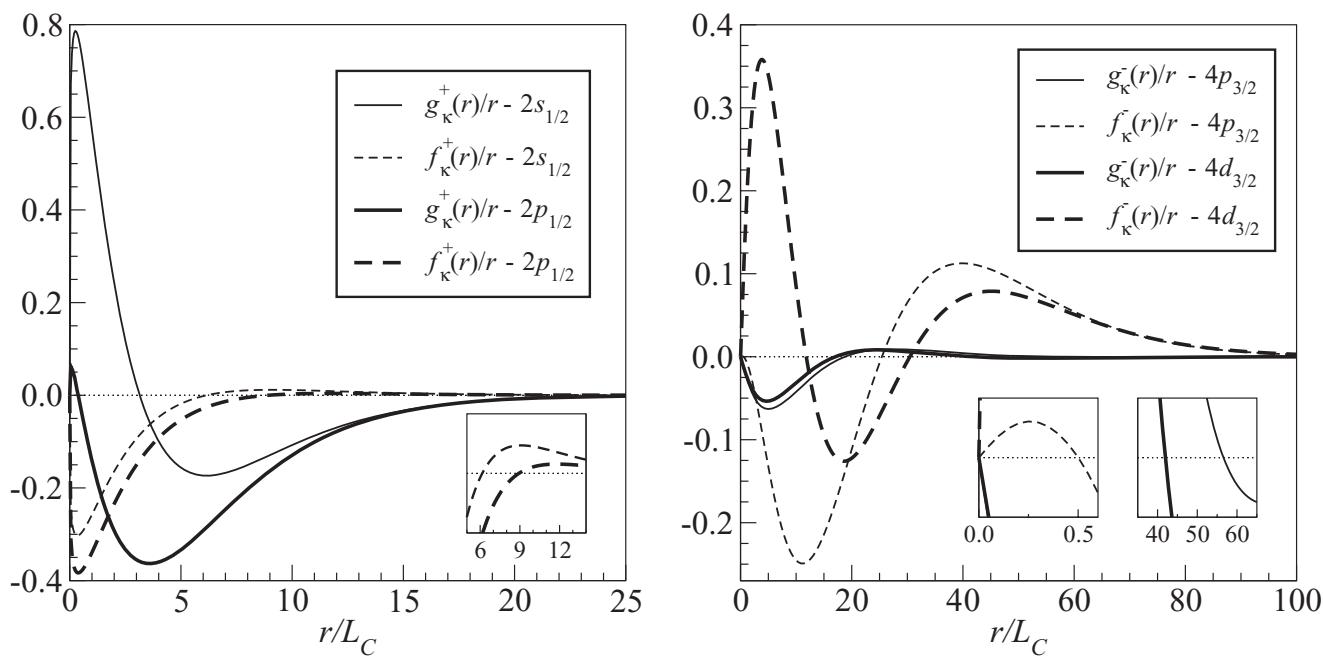

FIG. 1. Normalized radial wave functions $g_{\kappa}(r) / r$ and $f_{\kappa}(r) / r$ for the $E^{+}$states $2 s_{1 / 2}, 2 p_{1 / 2}\left(\kappa=\mp 1, n_{r}=1, n_{g, f}=2\right)$ and the $E^{-}$states $4 p_{3 / 2}, 4 d_{3 / 2}\left(\kappa=\mp 2, n_{r}=2, n_{g, f}=3\right)$, when $\alpha_{\Delta}=0.5$ and $\alpha_{\Sigma}=-0.8$. The radial wave functions were scaled in order to be dimensionless. $L_{C}=\hbar /(m c)$ is the Compton wavelength.

From the values at the origin of associated Laguerre polynomials (see, for instance, Ref. [17]) one has

$$
\frac{L_{n_{r}}^{2 \gamma}(0)}{L_{n_{r}-1}^{2 \gamma}(0)}=\frac{n_{r}+2 \gamma}{n_{r}} .
$$

Since there is an extra node at $r=\rho=0$ and the equality in (37) corresponds to a node at the origin, from Eqs. (34) and (35) one may write

$$
\begin{aligned}
& n_{g}= \begin{cases}n_{r}, & \frac{2 \gamma+n_{r}}{\kappa+\eta_{2}} \leqslant-\frac{n_{r}+2 \gamma}{n_{r}} \\
n_{r}+1, & \frac{2 \gamma+n_{r}}{\kappa+n_{2}}>-\frac{n_{r}+2 \gamma}{n_{r}}\end{cases} \\
& n_{f}=\left\{\begin{array}{ll}
n_{r}, & -\frac{2 \gamma+n_{r}}{\kappa+\eta_{2}} \leqslant-\frac{n_{r}+2 \gamma}{n_{r}} \\
n_{r}+1, & -\frac{2 \gamma+n_{r}}{\kappa+\eta_{2}}>-\frac{n_{r}+2 \gamma}{n_{r}}
\end{array} .\right.
\end{aligned}
$$

where $\operatorname{sgn}(x)$ is the signum function giving the sign of $x$.

On the other hand, from (15) and (25) and when $\alpha_{\Sigma} \alpha_{\Delta}<0$, the following inequalities hold:

$$
\left|\eta_{2}\right|>n_{r} \quad \gamma>|\kappa| \Rightarrow\left|\eta_{2}\right|>n_{r}+|\kappa|
$$

meaning that

$$
\left|\kappa+\eta_{2}\right|>n_{r},
$$

which is true when $\kappa$ and $\eta_{2}$ have the either the same or different signs, which is to say that it is true for any sign of $\kappa$ and $\eta_{2}$. From (39) and (40) it follows that, when $\alpha_{\Sigma} \alpha_{\Delta}<0$, one has

$$
\begin{aligned}
& n_{g}=n_{r}+1 \\
& n_{f}=n_{r}+1
\end{aligned}
$$

for any $\kappa$.
Note that this result applies to both $E^{+}$and $E^{-}$solutions. Also, since $\alpha_{\Sigma}$ and $\alpha_{\Delta}$ have different signs, $\gamma>1$ and thus $g_{\kappa}(r) / r$ and $f_{\kappa}(r) / r$ have the same nodes as $g_{\kappa}(r)$ and $f_{\kappa}(r)$.

In Fig. 1 this is shown explicitly for the $E^{+}$levels $2 s_{1 / 2}, 2 p_{1 / 2}\left(\kappa=\mp 1, n_{r}=1\right)$ (left panel) and for the $E^{-}$levels $4 p_{3 / 2}, 4 d_{3 / 2}\left(\kappa=\mp 2, n_{r}=2\right)$ (right panel), for $\alpha_{\Delta}=0.5$ and $\alpha_{\Sigma}=-0.8$. The radial coordinate is given in units of the Compton wavelength $L_{C}=\hbar /(m c)$. Here one uses, as usual, the spectroscopic notation $n \ell_{j}$ referring to the upper component of the Dirac spinor.

It is interesting to remark that this node structure does not follow the rule for the Dirac equation with vector and scalar radial potentials derived by Leviatan and Ginocchio in Ref. [18],

$$
n_{f}=\left\{\begin{array}{ll}
n_{g}, & \kappa<0 \\
n_{g}+1, & \kappa>0
\end{array},\right.
$$

by which only for negative $\kappa$ the upper radial and lower radial functions of the Dirac spinor have the same number of nodes. The structure (44) also holds for antiparticles in Woods-Saxon scalar and vector mean-field potentials, as shown in Refs. [3,6]. This may be because these potentials are finite at $r=0$, as are the nuclear mean-field scalar and vector potentials, and thus the behavior of the respective radial functions at the origin is different from the present case of Coulomb potentials.

\section{SPIN AND PSEUDOSPIN SYMMETRIES IN THE DIRAC HAMILTONIAN WITH SCALAR AND VECTOR COULOMB POTENTIALS}

To study the conditions under which the Dirac Hamiltonian (5) with the Coulomb potentials (6) and (7) has bound solutions near the conditions of spin and pseudospin symmetry, we will perform an expansion of Eq. (26) for small $\alpha_{\Delta}$ (spin) and $\alpha_{\Sigma}$ (pseudospin). 
If we expand (26) (using the principal quantum number $n$ ) until next-to-next-to-leading order in $\alpha_{\Delta}$ one gets

$$
\begin{gathered}
E_{n, \kappa}^{+}=m c^{2}\left\{1-\frac{2 \alpha_{\Sigma}^{2}}{\alpha_{\Sigma}^{2}+4 n^{2}}+\frac{4 \alpha_{\Sigma}^{3}\left(\kappa^{2}-2 n|\kappa|\right)}{\kappa^{2}\left(\alpha_{\Sigma}^{2}+4 n^{2}\right)^{2}} \alpha_{\Delta}\right. \\
\left.-\frac{\alpha_{\Sigma}^{4}\left\{4 n^{3}|\kappa|\left[\alpha_{\Sigma}^{2}+4\left(n^{2}-4 \kappa^{2}\right)\right]+\kappa^{2}\left[\alpha_{\Sigma}^{2}\left(\kappa^{2}-4 n^{2}\right)+20 \kappa^{2} n^{2}+48 n^{4}\right]\right\}}{2 \kappa^{4} n^{2}\left(\alpha_{\Sigma}^{2}+4 n^{2}\right)^{3}} \alpha_{\Delta}^{2}+O\left(\alpha_{\Delta}^{3}\right)\right\} \\
E_{n, \kappa}^{-}=m c^{2}\left[-1+\frac{\alpha_{\Delta}^{2}}{2 n^{2}}-\frac{\alpha_{\Sigma}\left(\kappa^{2}-2 n|\kappa|\right)}{4 \kappa^{2} n^{4}} \alpha_{\Delta}^{3}+O\left(\alpha_{\Delta}^{4}\right)\right],
\end{gathered}
$$

while for $\alpha_{\Sigma}$ one has

$$
\begin{gathered}
E_{n, \kappa}^{+}=m c^{2}\left[1-\frac{\alpha_{\Sigma}^{2}}{2 n^{2}}+\frac{\alpha_{\Delta}\left(\kappa^{2}-2 n|\kappa|\right)}{4 \kappa^{2} n^{4}} \alpha_{\Sigma}^{3}+O\left(\alpha_{\Sigma}^{4}\right)\right] \\
E_{n, \kappa}^{-}=m c^{2}\left\{-1+\frac{2 \alpha_{\Delta}^{2}}{\alpha_{\Delta}^{2}+4 n^{2}}-\frac{4 \alpha_{\Delta}^{3}\left(\kappa^{2}-2 n|\kappa|\right)}{\kappa^{2}\left(\alpha_{\Delta}^{2}+4 n^{2}\right)^{2}} \alpha_{\Sigma}\right. \\
\left.+\frac{\alpha_{\Delta}^{4}\left\{4 n^{3}|\kappa|\left[\alpha_{\Delta}^{2}+4\left(n^{2}-4 \kappa^{2}\right)\right]+\kappa^{2}\left[\alpha_{\Delta}^{2}\left(\kappa^{2}-4 n^{2}\right)+20 \kappa^{2} n^{2}+48 n^{4}\right]\right\}}{2 \kappa^{4} n^{2}\left(\alpha_{\Delta}^{2}+4 n^{2}\right)^{3}} \alpha_{\Sigma}^{2}+O\left(\alpha_{\Sigma}^{3}\right)\right\}
\end{gathered}
$$

From these equations is clear that $E^{+(-)}$is the energy of bound positive (negative) energy states for small $\alpha_{\Delta}\left(\alpha_{\Sigma}\right)$. The symmetry of the expressions above when one switches from $\alpha_{\Delta}$ to $\alpha_{\Sigma}$ and from positive to negative energy states is just the illustration of the charge conjugation transformations as given by Eq. (27). From the equations above one sees also that when the zeroth-order term is just $\pm m c^{2}$ the respective symmetry cannot be realized, since there are no bound states. For positive energy states one sees this in Eq. (47) (pseudospin symmetry) and for negative energy states in Eq. (46) (spin symmetry). Again, this is consistent to what is known from realistic calculations in nuclei, since $\Sigma$ and $-\Delta$ potentials act as binding potentials for fermions and antifermions, respectively $[3,6]$.

It is interesting to examine how the spin and pseudospin symmetries get broken and the corresponding energy splittings of the (pseudo)spin partners.

Equations (45)-(48) reveal that the breaking of spin symmetry is perturbative and the breaking of pseudosymmetry is nonperturbative for $E^{+}$states. The reverse is true for $E^{-}$states. This can be seen from the fact that $\alpha_{\Delta}$ and $\alpha_{\Sigma}$ act as perturbative parameters respectively in Eqs. (45) and (48) so that, as these parameters go to zero, one can go continuously to a bound state (the nonperturbed state). This does not happen for the expansions of $E^{+}$in terms of $\alpha_{\Sigma}$, which would lead to pseudospin symmetry in the limit $\alpha_{\Sigma} \rightarrow 0$, and of $E^{-}$in terms of $\alpha_{\Delta}$, leading to spin symmetry.

In previous works in which the perturbative nature of pseudospin (or spin in the case of antifermions) was related to the onset of the respective symmetry, one would either examine the effect the pseudospin-orbit or spin-orbit term on the energy splittings of the respective doublets $[6,8,9]$ or analyze the convergence of a perturbation expansion in the respective potential strength [10]. In Ref. [6] one was able to show that in the limit of vanishing $\Delta$ (spin) or $\Sigma$ (pseudospin) for nucleon and antinucleons respectively those spin-orbit or pseudospin-orbit terms fully account for the splitting of spin or pseudospin doublets. These terms can be obtained from the second-order radial equations for $g_{\kappa}(r)$ and $f_{\kappa}(r)$, respectively (see, for instance, Ref. [9])

$(\hbar c)^{2}\left[\frac{1}{r^{2}} \frac{d}{d r}\left(r^{2} \frac{d}{d r}\right)-\frac{\kappa(\kappa+1)}{r^{2}}+\frac{\Delta^{\prime}}{E+m c^{2}-\Delta(r)}\left(\frac{d}{d r}+\frac{1+\kappa}{r}\right)\right] \frac{g_{\kappa}(r)}{r}=-\left[E-m c^{2}-\Sigma(r)\right]\left[E+m c^{2}-\Delta(r)\right] \frac{g_{\kappa}(r)}{r}$,

$(\hbar c)^{2}\left[\frac{1}{r^{2}} \frac{d}{d r}\left(r^{2} \frac{d}{d r}\right)-\frac{\kappa(\kappa-1)}{r^{2}}+\frac{\Sigma^{\prime}}{E-m c^{2}-\Sigma(r)}\left(\frac{d}{d r}+\frac{1-\kappa}{r}\right)\right] \frac{f_{\kappa}(r)}{r}=-\left[E-m c^{2}-\Sigma(r)\right]\left[E+m c^{2}-\Delta(r)\right] \frac{f_{\kappa}(r)}{r}$,

where the prime means derivative with respect to $r$. These are proportional to the derivatives of $\Delta$ (spin) and $\Sigma$ (pseudospin) and thus, for small enough values of $\alpha_{\Delta}$ and $\alpha_{\Sigma}$, respectively, their expectation values contributing to the energy splitting of spin and pseudospin partners should be linear in those parameters. In particular, one may relate the contributions from spin-orbit and pseudo-orbit couplings to the total energy to the following terms coming from Eqs. (49) and (50) as was done in Ref. [9]:

$$
\begin{aligned}
E^{\mathrm{SO}}= & -(\hbar c)^{2} \int_{0}^{\infty} \frac{\Delta^{\prime}}{\left(E+m c^{2}-\Delta\right)^{2}} \\
& \times \frac{1+\kappa}{r} g_{\kappa}^{2} \mathrm{~d} r /\left(\int_{0}^{\infty} g_{\kappa}^{2} \mathrm{~d} r\right)
\end{aligned}
$$




$$
\begin{aligned}
E^{\mathrm{PSO}}= & -(\hbar c)^{2} \int_{0}^{\infty} \frac{\Sigma^{\prime}}{\left(E-m c^{2}-\Sigma\right)\left(E+m c^{2}-\Delta\right)} \\
& \times \frac{1-\kappa}{r} f_{\kappa}^{2} \mathrm{~d} r /\left(\int_{0}^{\infty} f_{\kappa}^{2} \mathrm{~d} r\right) .
\end{aligned}
$$

These terms are consistent with the nonrelativistic formula for spin-orbit coupling, the first one applied to positive energy states and the second one to negative energy states. The origin of the factors $1+\kappa$ for the upper component and to $1-\kappa$ for the lower component can be traced to the action of the operator $\boldsymbol{L} \cdot \boldsymbol{S}$ upon the spinor (9), since $\boldsymbol{L} \cdot \boldsymbol{S} \psi=-\hbar^{2} / 2(\beta K+I) \psi$ with $K \psi=\kappa \psi$ and $\boldsymbol{S}=\hbar / 2 \boldsymbol{\Sigma}$.

One may note that the next-to-leading order terms in Eqs. (45) and (47) are of first order in $\alpha_{\Delta}$ and $\alpha_{\Sigma}$ respectively. Since those terms are responsible, when potentials go to zero, for breaking spin and pseudospin symmetries, respectively, one could associate them with the spin-orbit and pseudospinorbit coupling terms as was done with Woods-Saxon-type potentials $[6,9]$. However, contrary to what happens with those potentials, in the present case, because of the functional form of Coulomb potentials, the spin-orbit term (51) has a double pole at $r=\hbar c \alpha_{\Delta} /\left(E+m c^{2}\right)$, so it cannot be calculated separately from the so-called Darwin term, coming from the derivative term in Eq. (49) and also multiplied to $\Delta^{\prime}$. The sum of the two terms must of course be finite, but this means that one cannot explicitly calculate the contribution of the spin orbit from (51) in the Coulomb case. A similar thing happens for the negative energy case, for which it was possible to calculate separately the pseudospin-orbit contribution with Woods-Saxon-type potentials [6] but not in the present case due to the pole structure of the integrand in (52) for general values of $\alpha_{\Delta}$ and $\alpha_{\Sigma}$.

In spite of this, we may state that, for vector and scalar Coulomb potentials, one can establish a connection between the realization of exact pseudospin and spin symmetries for bound states and their perturbative breaking as was shown to be the case for nuclear mean-field potentials.

\section{A. Quantum numbers for exact spin and pseudospin symmetries}

Finally, we discuss the quantum numbers of states when there is either exact spin or pseudospin symmetries (i.e., when $\alpha_{\Delta}=0$ or $\alpha_{\Sigma}=0$, respectively). The energies of those levels are given by

$$
\begin{gathered}
E_{n, \kappa}^{+}=m c^{2}\left(1-\frac{2 \alpha_{\Sigma}^{2}}{\alpha_{\Sigma}^{2}+4 n^{2}}\right) \quad(\text { spin }), \\
E_{n, \kappa}^{-}=m c^{2}\left(-1+\frac{2 \alpha_{\Delta}^{2}}{\alpha_{\Delta}^{2}+4 n^{2}}\right) \quad \text { (pseudospin). }
\end{gathered}
$$

One may notice immediately that these energies only depend on the principal quantum number $n$. This situation looks very similar to what happens with the energy levels of a nonrelativistic hydrogenic atom. To see how it comes about we start out with the spin symmetry case $\left(\Delta=0\right.$ or $\left.\alpha_{\Delta}=0\right)$. The corresponding Dirac equation reads

$$
\left[\boldsymbol{\alpha} \cdot \boldsymbol{p} c+\beta m c^{2}+\frac{1}{2}(I+\beta) \Sigma\right] \psi=E \psi,
$$

with $\Sigma=\alpha_{\Sigma} \hbar c / r$.
Following the procedure of Ref. [19] we use the projectors $\psi_{ \pm}=P_{ \pm} \psi=[(I \pm \beta) / 2] \psi$ such that

$$
\psi_{+}=\left(\begin{array}{l}
\phi \\
0
\end{array}\right) \quad \psi_{-}=\left(\begin{array}{l}
0 \\
\chi
\end{array}\right),
$$

where $\phi$ and $\chi$ are respectively the upper and lower twocomponent spinors. Using the properties and anticommutation relations of the matrices $\beta$ and $\alpha$ we can apply the projectors $P_{ \pm}$to the Dirac equation (55) and decompose it into two coupled equations for $\psi_{+}$and $\psi_{-}$,

$$
\begin{gathered}
c \boldsymbol{\alpha} \cdot \boldsymbol{p} \psi_{-}+\left(m c^{2}+\Sigma\right) \psi_{+}=E \psi_{+} \\
c \boldsymbol{\alpha} \cdot \boldsymbol{p} \psi_{+}-m c^{2} \psi_{-}=E \psi_{-} .
\end{gathered}
$$

From this last equation we get $\psi_{-}$and replace it in (57) to give

$$
p^{2} c^{2} \psi_{+}=\left(E+m c^{2}\right)\left(E-m c^{2}-\Sigma\right) \psi_{+} .
$$

or yet

$$
\frac{p^{2}}{2 m} \psi_{+}=\left(\mathcal{E}^{\prime}-\Sigma^{\prime}\right) \psi_{+}
$$

where

$$
\begin{aligned}
\mathcal{E}^{\prime} & =\left(\frac{\mathcal{E}}{2 m c^{2}}+1\right) \mathcal{E}, \quad \Sigma^{\prime}=\left(\frac{\mathcal{E}}{2 m c^{2}}+1\right) \Sigma, \\
\mathcal{E} & =E-m c^{2}
\end{aligned}
$$

Equation (60) is just the Schrödinger equation for a hydrogenic atom of "atomic number"

$$
Z^{\prime}=-\left(\frac{\mathcal{E}}{2 m c^{2}}+1\right) \alpha_{\Sigma} / \alpha
$$

where again $\alpha$ is the fine structure constant. The energy of this "atom" is just [see Eq. (31)]

$$
\mathcal{E}^{\prime}=-m c^{2}\left(\frac{\mathcal{E}}{2 m c^{2}}+1\right)^{2} \frac{\alpha_{\Sigma}^{2}}{n^{2}} .
$$

Replacing the expressions (61) into this last equation one gets Eq. (53).

The fact that the energy only depends on $n$ in spin symmetry conditions implies also that there is an extra level degeneracy. Because of the relation $n=n_{r}+|\kappa|$, levels with the same principal quantum number and $|\kappa| \leqslant n$ are degenerate. This also has a correspondence to the well-known degeneracy of levels of a hydrogenic atom in which all levels with $\ell \leqslant n-$ 1 and the same $n$ are degenerate. These properties can be traced back to the fact that in spin symmetry conditions and radial potentials there is an $S U(2)$ symmetry generated by the operator

$$
\mathcal{L}=\boldsymbol{L} P_{+}+\frac{1}{p^{2}} \boldsymbol{\alpha} \cdot \boldsymbol{p} \boldsymbol{L} \boldsymbol{\alpha} \cdot \boldsymbol{p} P_{-}=\left(\begin{array}{cc}
\boldsymbol{L} & 0 \\
0 & U_{p} \boldsymbol{L} U_{p}
\end{array}\right),
$$

where $U_{p}=\boldsymbol{\sigma} \cdot \boldsymbol{p} /\left(\sqrt{p^{2}}\right)$ is the helicity operator. This means that the orbital angular momentum $\ell$ of the upper component of the Dirac spinor is a good quantum number in this case [1,19]. Another way of stating this is saying that when there is spin symmetry there is no spin-orbit coupling.

A similar reasoning can be made when there is pseudospin symmetry $\left(\Sigma=0, \alpha_{\Sigma}=0\right)$. In this case one would get the 
following equation for the lower component $\psi_{-}$:

$$
\frac{\boldsymbol{p}^{2}}{2 m} \psi_{-}=\left(\mathcal{E}^{\prime}-\Delta^{\prime}\right) \psi_{-},
$$

where

$$
\begin{aligned}
\mathcal{E}^{\prime} & =\left(\frac{\mathcal{E}}{2 m c^{2}}+1\right) \mathcal{E}, \quad \Delta^{\prime}=-\left(\frac{\mathcal{E}}{2 m c^{2}}+1\right) \Delta, \\
\mathcal{E} & =-E-m c^{2} .
\end{aligned}
$$

As one would expect for negative energy states and from charge conjugation, this amounts basically to change the energy sign and also replacing $\Sigma$ by $-\Delta$ [6]. The SU(2) generator would be in this case

$\tilde{\mathcal{L}}=\frac{1}{p^{2}} \boldsymbol{\alpha} \cdot \boldsymbol{p} \boldsymbol{L} \boldsymbol{\alpha} \cdot \boldsymbol{p} P_{+}+\boldsymbol{L} P_{-}=\left(\begin{array}{cc}U_{p} \boldsymbol{L} U_{p} & 0 \\ 0 & \boldsymbol{L}\end{array}\right)$.

and in this case the orbital angular momentum $\tilde{\ell}$ of the lower component of the Dirac spinor is a good quantum number $[1,19]$.

\section{CONCLUSION}

We analyzed in detail the analytical solutions of Dirac equation with scalar $S$ and vector $V$ Coulomb radial potentials in terms of the sum $\Sigma$ and difference $\Delta$ potentials. Besides reviewing the solutions at this light, we established the node structure of their radial solutions and examined the solutions near the limit of spin and pseudospin symmetries (i.e., when $\Delta=0$ and $\Sigma=0$, respectively). We assessed the perturbative nature of those symmetries, and confirmed previous results with other types of potentials, namely, that spin symmetry is perturbative and pseudospin symmetry is nonperturbative for positive energy solutions, while the reverse happens for negative energy solutions. We also found that in conditions of exact spin and pseudospin symmetries the solutions of the Dirac equation have similar features of the well-known nonrelativistic solutions for hydrogenic atoms, namely that they depend only on the principal quantum number and exhibit a similar degeneracy.

\section{ACKNOWLEDGMENTS}

We acknowledge financial support from Conselho Nacional de Desenvolvimento Científico e Tecnológico (CNPq) and Quadro de Referência Estratégico Nacional/Fundo Europeu de Desenvolvimento Regional (QREN/FEDER), the Programme COMPETE, under Project No. PTDC/FIS/113292/2009. P.A. would like also to thank the Universidade Estadual Paulista, Guaratinguetá Campus, for supporting his stays in its Physics and Chemistry Department.
[1] J. N. Ginocchio, Phys. Rep. 414, 165 (2005).

[2] B.-N. Lu, E.-G. Zhao, and S.-G. Zhou, Phys. Rev. Lett. 109, 072501 (2012)

[3] S.-G. Zhou, J. Meng, and P. Ring, Phys. Rev. Lett. 91, 262501 (2003).

[4] X. T. He, S. G. Zhou, J. Meng, E. G. Zhao, and W. Scheid, Eur. Phys. J. A 28, 265 (2006).

[5] H. Liang, W- H. Long, J. Meng, and N. V. Giai, Eur. Phys. J. A 44, 119 (2010).

[6] R. Lisboa, M. Malheiro, P. Alberto, M. Fiolhais, and A. S. de Castro, Phys. Rev. C 81, 064324 (2010).

[7] C.-Y. Song, J.-M. Yao, and J. Meng, Chin. Phys. Lett. 26, 122102 (2009); C.-Y. Song and J.-M. Yao, Chin. Phys. C. 34, 1425 (2010); C.-Y. Song, J.-M. Yao, and J. Meng, Chin. Phys. Lett. 28, 092101 (2011).

[8] S. Marcos, L. N. Savushkin, M. López-Quelle, and P. Ring, Phys. Rev. C 62, 054309 (2000).

[9] P. Alberto, M. Fiolhais, M. Malheiro, A. Delfino, and M. Chiapparini, Phys. Rev. C 65, 034307 (2002).
[10] H. Liang, P. Zhao, Y. Zhang, J. Meng, and N. V. Giai, Phys. Rev. C 83, 041301(R) (2011).

[11] R. Lisboa, M. Malheiro, A. S. de Castro, P. Alberto, and M. Fiolhais, Phys. Rev. C 69, 024319 (2004); A. S. de Castro, P. Alberto, R. Lisboa, and M. Malheiro, ibid. 73, 054309 (2006).

[12] G. Soff, B. Müller, J. Rafelski, and W. Greiner, Z. Naturforsch. 28a, 1389 (1973).

[13] S. Zarrinkamar, H. Hassanabadi, and A. A. Rajabi, Int. J. Mod. Phys. A 26, 1011 (2011).

[14] W. Greiner, B. Müller, and J. Rafelski, Quantum Electrodynamics of Strong Fields (Springer-Verlag, New York, 1985).

[15] A. Leviatan, Phys. Rev. Lett. 92, 202501 (2004).

[16] M. H. Johnson and B. A. Lippmann, Phys. Rev. 78, 329 (1950).

[17] Handbook of Mathematical Functions, edited by M. Abramowitz and I. Stegun (Dover Publications, New York, 1972).

[18] A. Leviatan and J. N. Ginocchio, Phys. Lett. B 518, 214 (2001).

[19] P. Alberto, A. S. de Castro, and M. Malheiro, Phys. Rev. C 75, 047303 (2007). 